\title{
Effect of Maternal Food Restriction on the Uterus of Female Rats from the First and Second Generation
}

\author{
Saleh H. Alwasel \\ Department of Zoology, College of Science, King Saud University, Riyadh, Kingdom of Saudi Arabia \\ Email: salwasel@ksu.edu.sa
}

Received 10 March 2016; accepted 27 March 2016; published 31 March 2016

Copyright (C) 2016 by author and Scientific Research Publishing Inc. This work is licensed under the Creative Commons Attribution International License (CC BY). http://creativecommons.org/licenses/by/4.0/ c) (7) Open Access

\begin{abstract}
Postnatal life is affected by changes in fetal development due to adverse events. The present study examined the effect of maternal food restriction on uterine structure and capacity in female rats from the first and second generations (FR1 and FR2) before and after puberty. Pregnant rats were fed ad libitum (Control, C) or maintained under food-restriction conditions (50\%FR) during pregnancy. After delivery, all groups were given free access to food. Females from the first generation (FR1) were divided into two subgroups for either uterine dissection or to produce the next generation (FR2). Similar to FR1, FR2 females were exposed to maternal food restriction following the same procedure. FR2 females were also assigned into two subgroups for either uterine dissection or for mating to examine pregnancy outcomes. Uteri from the C, FR1 and FR2 groups were dissected at 4 weeks of age (before puberty) or at 8 weeks of age (after puberty) to evaluate uterine weight and uterine horn length. Tissues were stained with eosin and haematoxylin for examination vialight microscopy. The results showed that food restriction did not affect uterine weight or uterine horn length in FR1 and FR2 females before puberty. However, there were effects of maternal food restriction on uterine weight and length at 8 weeks of age. Uterine weight and uterine horn length were significantly reduced in the FR1 and FR2 groups compared with the values of the controls. This was associated with changes in pregnancy outcomes. Litter size was not affected in the FR1 group; however, litter weight was significantly reduced, leading to an $18 \%$ reduction in pup weight at birth. Unlike with FR1 litters, the size of FR2 litters was significantly reduced, leading to a lower degree of pup body weight reduction at birth (only 7\%). The gestational length was normal in FR1 females but was significantly decreased in FR2 females. No histopathological changes were observed. These results showed that the uterus was affected by maternal food restriction. It seemed that FR2 females had a different reproductive strategy: they tended to reduce litter size to maintain pup weight at birth. In conclusion, maternal food restriction affects the uteri of the first and second generation. Females from the FR1 and FR2 groups followed different reproductive strategies according to their previous experience with exposure to intrauterine food
\end{abstract}




\section{restriction.}

\section{Keywords}

\section{Food Restriction, Pregnancy Outcome, Uterus Weight, Uterine Horn Height}

\section{Introduction}

It has been widely reported that early fetal development is a critical determinant of adult physiology [1]. Indeed, several adulthood diseases can be attributed to an adverse embryonic environment, such as undernutrition, which may alter the structure of specific fetal organs [2]-[5]. These changes in organ structures are permanent and lead to functional deficiencies [6]. Extensive studies have shown that maternal malnutrition can change the structure and function of the kidney, leading to hypertension [7]-[9]. Only a few studies on nutrition in relation toanimal fertility have been carried out [10] [11], and they suggest that food deprivation may increase the risk of infertility. Moreover, it has been shown in animal models that maternal diet during gestation can increase the risk of breast cancer in offspring [12]-[14]. It has also been postulated that ovarian cancer is initiated by the exposure of the fetal ovaries to maternal sex hormones, which may be the product of poor nutrition and growth in early childhood, followed by catch-up pre-pubertal growth [15].

Despite a large body of evidence that maternal undernutrition programmes various organs in the offspring [1]-[4], little is known about the effects of maternal food restriction on the reproductive system in female offspring over two generations [16] [17]. The aim of the present study was to investigate the effect of maternal food restriction during pregnancy on the structure and capacity of the uterus of female rats from the first and second generations.

\section{Material and Methods}

\subsection{Experimental Design}

Ten-week old virgin Wistar female rats weighing $230 \pm 20$ g were obtained from The Animal Unit, King Saud University. All experiments carried out in the current study were performed in accordance with the guidelines of The Research Ethics Committee in King Saud University. Animals were housed individually for three days at a constant temperature and humidity. They were given a free access to tap water and standard laboratory chow (23\% protein, 4.5\% fat, $3030 \mathrm{kcal} / \mathrm{kg}$; lab diet 5001, Brentwood, MO). Mating with virgin males was confirmed by the presence of a white sperm plug. Pregnant females were randomly assigned into one of two groups. The first was the control group (C, $n=20)$, which received ad libitum food from conception until they gave birth. The second was the 50\% food-restricted group ( $50 \% \mathrm{FR}, n=20$ ), which received only half of ad libitum food throughout gestation. At birth, all groups were given free access to water and food. Litter size and litter weight were recorded at birth. Average pup weight was calculated as litter weight divided by litter size. The number of pups per mother was reduced to 4 males and 4 females to allow equal chances for suckling as female rats have only 8 nipples. After a complete weaning, male offspring were removed and female offspring were divided into two subgroups. One subgroup was analysed as the first generation (FR1) and the other subgroup was mated to produce the second generation (FR2). Mothers of the F2 generation were treated exactly the same as the mothers of the F1 generation. F2 litter sizes and weights were recorded, and litter sizes were reduced to 4 males and 4 females per mother. Half of the F2 females were mated at the same age as the other groups to examine their litter sizes and litter weights in addition to their gestational length.

Females from the F1 and F2 generations were humanely killed at 4 (before puberty) or 8 (after puberty) weeks of age to collect the uteri. This allowed the investigator to examine the degree of structural alteration in the uteri of the F1 females, which were exposed to maternal food restriction once, and F2 females, which were exposed to maternal food restriction twice. The uterus weight $(\mathrm{g})$ and uterine horn length $(\mathrm{mm})$ were recorded in both generations at 4 and 8 weeks of age.

Uteri were immediately fixed in $10 \%$ neutral paraformaldehyde at room temperature for examination via light microscopy. Tissues were dehydrated using an ethanol gradient. Dehydrated tissues were cleared using xylene 
before being embedded in paraffin.

\subsection{Light Microscopy}

Tissue sections, 6 - $8 \mu \mathrm{m}$ thick, were prepared and stained with eosin and haematoxylin. Histological structures were examined, and the thickness of the uterine wall, muscular layer and endometrium layer were measured using image management software (MoticDigiLabII-Digital Laboratory Network version).

\subsection{Data Analysis}

Data are presented as the mean \pm standard error. Differences between groups were examined using one-way ANOVA followed by post hoc tests. Analyses were performed using SPSS software version 16. Differences between means were considered statically significant when the $p$ value was less than 0.05

\section{Results}

\subsection{Effect of Maternal 50\%FR on Pregnancy Outcomes}

Gestational length was not affected by maternal food restriction in the first generation; however, it was significantly reduced in the second generation (Table 1).

Litter weight was significantly decreased in the FR1 and FR2 groups in comparison with the litter weight in the control group (Table 1). The litter size of FR1 rats was unaltered (10.03 \pm 0.38 , NS); however, litter size was significantly reduced in the FR2 group $(8.66 \pm 0.32, \mathrm{p}<0.05)$ compared with litter sizes in the control $(10.43 \pm 0.31)$ and FR1 groups. Birth weight was significantly reduced in the FR1 and FR2 group compared with birth weight in the controls $(C=6.24 \pm 0.12$, FR1 = $5.13 \pm 0.15, \mathrm{p}<0.05$, FR2 = $5.68 \pm 0.11 ; \mathrm{p}<0.05$ ); however, the degree of reduction was less pronounced in the FR2 group.

\subsection{Body Weight}

FR1 females were able to catch-up and significantly increase their body weight by 4 weeks of age relative to the controls, while FR2 females remained smaller than the other groups (Table 1). Similarly, FR1 females were significantly heavier than the control and FR2 females at 8 weeks of age, while the body weight of FR2 females was the lowest among all groups (Table 1).

\subsection{Uterine Indices}

At week four, the weight of the uterus was comparable between the three groups $(C=0.064 \pm 0.004$, FR1 $=$ $0.056 \pm 0.003$, FR2 $=0.054 \pm 0.004$; Figure 1 (a)). However, uterine weight was remarkably reduced in both FR1 and FR2 females in comparison with the control uteri at 8 weeks of age $(C=0.41 \pm 0.029$, FR1 $=0.28 \pm$ 0.014 , FR2 = $0.25 \pm 0.012$; Figure 1(b)).

Uterine horn length in 4-week-old offspring was not affected by maternal food restriction in either FR1 or FR2 females $(C=3.64 \pm 0.1$, FR1 $=3.075 \pm 0.15$, FR2 $=3.4 \pm 0.07$; Figure 2(a)). In contrast, uterine horn

Table 1. Effects of maternal food restriction on pregnancy outcomes and offspring body weight. * $=p<0.05$, $¥$ indicates that the difference between FR1 and FR2 was significant.

\begin{tabular}{cccc}
\hline Group & C & F1 & F2 \\
\hline Gestational length (day) & $21.75 \pm 0.09$ & $21.60 \pm 0.11$ & $20.82 \pm 0.13^{*}$ \\
Litter weight (g) & $63.89 \pm 1.84$ & $52.11 \pm 1.92^{*}$ & $47.83 \pm 1.54^{*}$ \\
Litter size (pups) & $10.43 \pm 0.31$ & $10.03 \pm 0.38$ & $8.66 \pm 0.32^{*}$ \\
Birth weight (g) & $6.24 \pm 0.12$ & $5.13 \pm 0.15^{*}$ & $5.78 \pm 0.11^{* \neq}$ \\
Body weight (g) at week 4 & $70.27 \pm 1.26$ & $75.72 \pm 1.26^{*}$ & $66.23 \pm 1.42^{* \neq}$ \\
Body weight (g) at week 8 & $114.41 \pm 2.47$ & $126.41 \pm 1.54^{*}$ & $103.27 \pm 1.94^{*} \neq$ \\
\hline
\end{tabular}




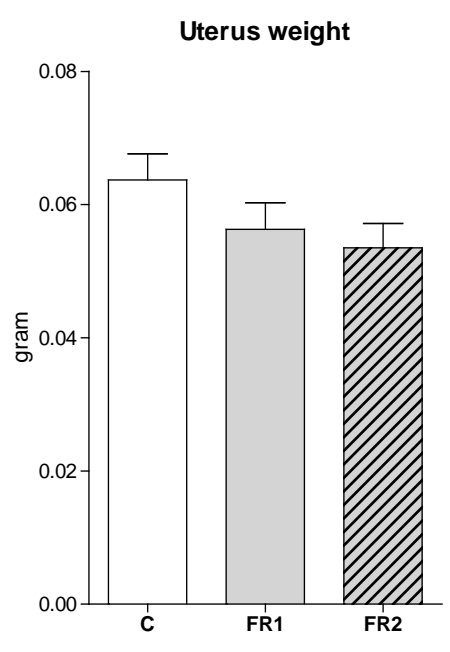

(a)

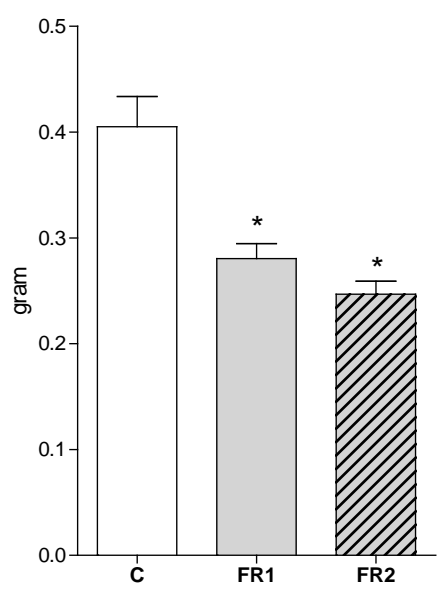

(b)

Figure 1. Effect of maternal food restriction on uterine weight at 4 (a) and 8 (b) weeks of age. $*=p<0.05$.

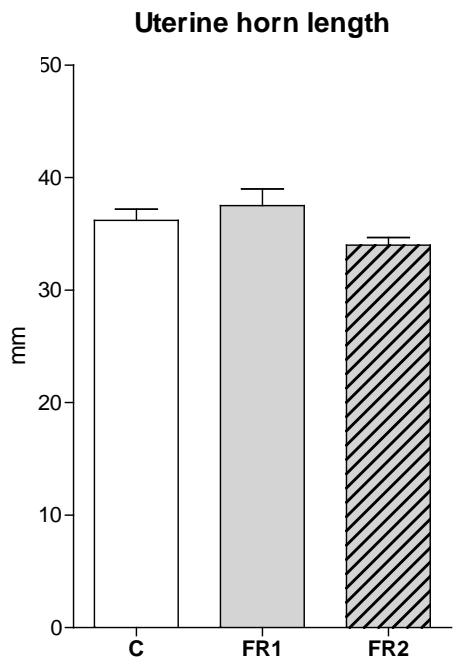

(a)

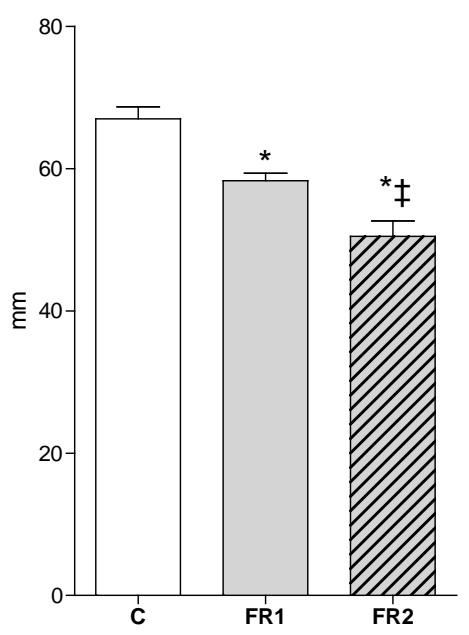

(b)

Figure 2. Effect of maternal food restriction on uterine horn length at week 4 (a) and 8 (b) weeks of age. $*=p<0.05, \ddagger$ indicates that the difference between FR1 and FR2 was significant.

length was significantly decreased in FR1 and FR2 females relative to the length in control off spring at 8 weeks of age. The observed effect was much stronger in FR2 females $(C=6.7 \pm 0.17$, FR1 $=5.8 \pm 0.10$, FR2 $=5.1 \pm$ 0.21 ; Figure 2(b)).

\subsection{Uterine Histology}

Light microscopy revealed no histopathological differences between the FR1, FR2 and control groups. Figure 3 shows representative histological images of uteri from the FR1, FR2 and control groups. Uterine wall thickness was similar in all groups at 4 weeks of age; however, uterine wall thickness tended to be thicker in the FR1 and FR2 groups than in the control group at weeks 8 of age, although the difference was not statically significant (Figure 4). To examine which of the main layers, namely the myometrium and endometrium, were increased, the investigator measured them separately. Figure 5 shows that the thickness of the myometrium (muscular layer) was not affected by maternal diet in FR1 and FR2 female offspring at all ages. In contrast, the thickness 


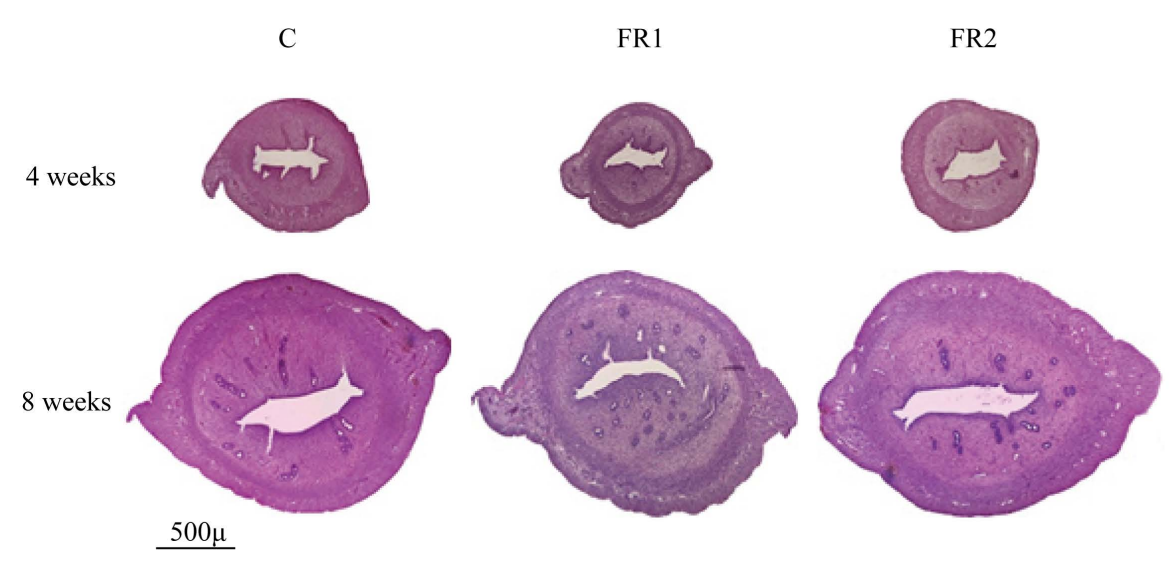

Figure 3. Representative cross sections of uteri from control (C), first generation (FR1) and second generation (FR2) females at 4 and 8 weeks of age. No histopathological changes between groups were observed.

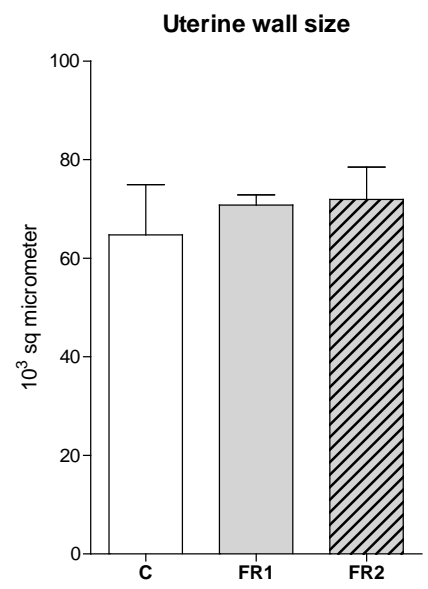

(a)

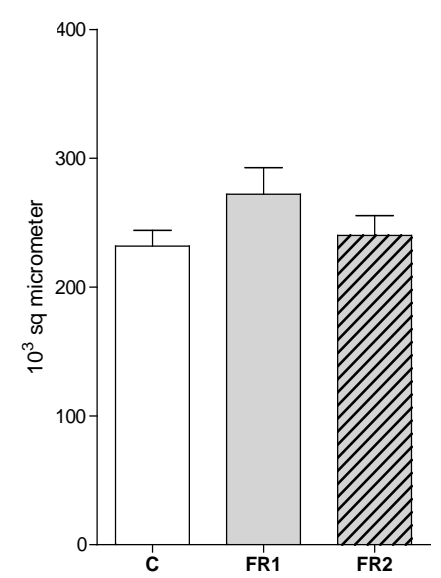

(b)

Figure 4. Uterine wall thickness was unaffected by maternal food restriction as measured at 4 and 8 weeks of age ((a) and (b), respectively).

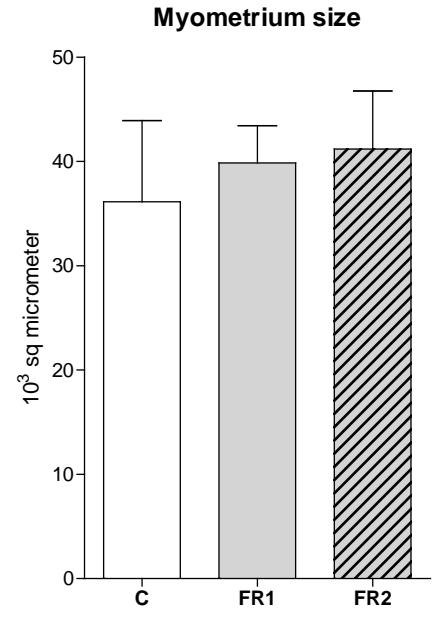

(a)

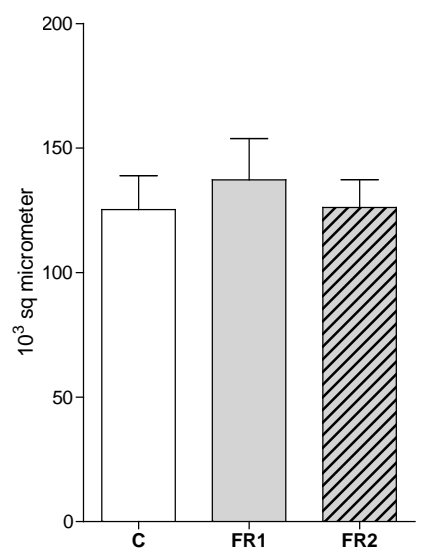

(b)

Figure 5. Effect of maternal food restriction on myometrial thickness. There were no significant differences between the groups at 4 (a) or 8 (b) weeks of age. 
of endometrium of FR1 was significantly greater than that of the other groups at 8 weeks of age but not at 4 weeks of age (Figure 6). The thickness of the endometrium of FR2 females was similar to the control group at 4 and 8 weeks of age.

\section{Discussion}

This study provides the first description of structural changes in the uteri of first and second generation females exposed to $50 \%$ maternal food restriction. It seems that female rats adapted to their previous nutritional conditions such that the reproductive strategies were different between the two generations exposed to $50 \%$ food restriction. Mothers of the FR1 group had the same fetal environment as the controls. Therefore, the strategy of mothers of the FR1 group was to preserve litter size at the expense of litter weight. This resulted in an 18\% reduction in pup weight at birth. In contrast, mothers of the FR2 group, which had experienced food restriction during their fetal development, seemed to have benefited from their previous experience. Accordingly, one remarkable change in their reproductive strategy was to reduce their litter sizes by $17 \%$ to preserve pup weight at birth. This resulted in a 7\%reduction in pup weight at birth in FR2 litters, which was much better than the reduction of 18\% observed in FR1 litters. Another change in the reproductive strategy observed in FR2 females was that the gestational duration was reduced by 22 hours, which is equivalent to $4.3 \%$ of the total gestation time in the control group. A linear regression analysis indicated that FR2 pups would have reached a normal weight if the gestational duration had been the same as in the control. It is not yet clear why the FR2 gestational duration was reduced, but one possible explanation is that the uterine space was too small, as shown in Figure 2. Another possible explanation is that a hormonal mechanism stimulated an early delivery, although this needs to be confirmed in future work.

The current results show that no significant differences in uterine weight or uterine horn length were present between the FR1 and FR2 groups at the pre-pubertal age (week four). However, effects of food restriction were observed at week 8, when uterine weight and uterine horn height were found to be significantly smaller in FR1 and FR2 females than in the control group. Week 4 corresponds to a pre-pubertal age and week 8 corresponds to an early adult age where sex hormones are active. The uterine cycle is correlated with the dynamics of female reproductive hormones, which prepare the uterus for embryo implantation [18]. It is known that female rats reach the age of puberty after week 4 (at approximately 30 days of age). At this age, a cascade of events, including hormonal release occurs. This includes the LH and FSH release that leads to ovarian maturation [19]. Because the female reproductive tract is inactive during the period from birth until the age of 30 days, we speculated that no significant differences in the size and weight of the uterus would be detected at week 4 between the FR1, FR2 and control groups. The significantly reduced uterine weight and uterine horn height ob-

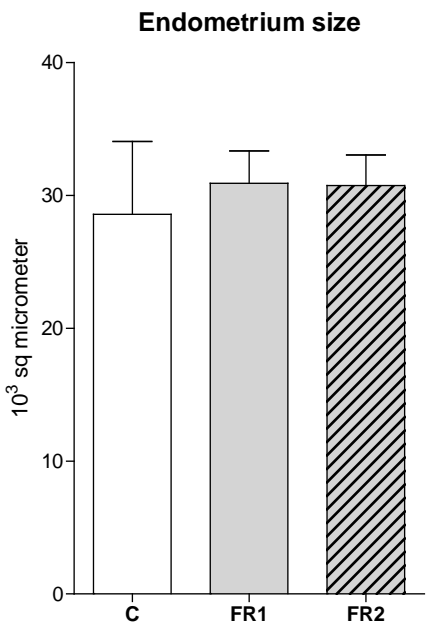

(a)

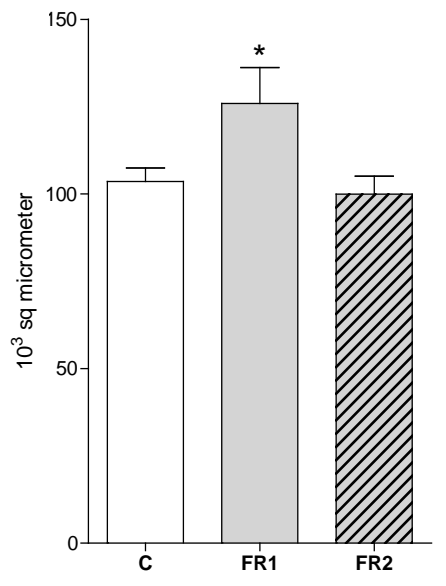

(b)

Figure 6. Endometrial thickness in C, FR1 and FR2 females at weeks 4 (a) 8 (b) weeks of age. The endometrium layer was thicker in FR1 females than in the other groups. $*=p<$ 0.05 . 
served in the FR1 and FR2 females relative to the control group at 8 weeks of age may be explained by differences in how sex hormones affected the development of the uterus in each group. Indeed, it has been hypothesized that food restriction affects reproductive development and hormonal levels by affecting the release of sex hormones, such as GnRH, LH and FSH [20] [21]. The concentration of estradiol or testosterone significantly decreases with the degree of food restriction [22]. This could also reduce the weight of the mammary glands [23] and may cause intrauterine growth restriction [24]. Moreover, it has been shown in several studies that maternal malnutrition can even affect the number of offspring; litters born to mothers under food restriction contain fewer offspring than those of the control groups [17] [25]. The quality of diet could also be a determining factor that affects the hormonal balance and, therefore, affects the reproductive tract of the mothers, potentially even affecting the sex ration of a population [26].

The histological examination of the specimens revealed that the thickness of the myometrium was similar in all groups. In contrast, the endometrium was increased in adult FR1 females, while the endometrium of FR2 females was comparable to that of the controls. Although a considerable effort was made to insure that the uterus was dissected in the same oestrus phase, these differences could be a result of hormonal activities. In future it would be better to include a subgroup of F1 that have been fed ad libitum during their pregnancy and investigate the uterus structure of their offspring (FR2 females that have been exposed to maternal food restriction once).

\section{Conclusion}

In summary, food restriction affected uterine weight and horn length in both FR1 and FR2 females at 8 weeks of age. This influenced the reproductive strategies in FR2 females and resulted in reduced litter sizes to preserve pups weight as much as possible. Nevertheless, FR2 pup body weight at birth was still smaller than in the control group because of the early delivery. This indicates that maternal food restriction has long-term consequences on the reproduction of subsequent generations.

\section{Acknowledgements}

The author would like to extend his sincere appreciation to the Academic Council of King Saud University for funding this research.

\section{References}

[1] Barker, D.J.P. (1990) The Fetal and Infant Origins of Adult Disease. British Medical Journal, 301, 1111-1111. http://dx.doi.org/10.1136/bmj.301.6761.1111

[2] Martin-Gronert, M.S. and Ozanne, S.E. (2006) Maternal Nutrition during Pregnancy and Health of the Offspring. Biochemical Society Transactions, 34, 779-782. http://dx.doi.org/10.1042/BST0340779

[3] Alwasel, S.H. and Ashton, N. (2009) Prenatal Programming of Renal Sodium Handling in the Rat. Clinical Science (London), 117, 75-84. http://dx.doi.org/10.1042/CS20080294

[4] Alwasel, S.H., Barker, D.J. and Ashton, N. (2012) Prenatal Programming of Renal Salt Wasting Resets Postnatal Salt Appetite, Which Drives Food Intake in the Rat. Clinical Science (London), 122, 281-288. http://dx.doi.org/10.1042/CS20110266

[5] Xu, G., Umezawa, M. and Takeda, K. (2009) Early Development Origins of Adult Disease Caused by Malnutrition and Environmental Chemical Substances. Journal of Health Science, 55, 11-19. http://dx.doi.org/10.1248/jhs.55.11

[6] Langley-Evans, S.C. (1999) Fetal Origins of Adult Disease. British Journal of Nutrition, 81, 5-6. http://dx.doi.org/10.1017/S0007114599000070

[7] Barker, D.J.P. (2001) The Malnourished Baby and Infant. British Medical Bulletin, 60, 69-88. http://dx.doi.org/10.1093/bmb/60.1.69

[8] Alwasel, S.H. and Ashton, N. (2012) Segmental Sodium Reabsorption by the Renal Tubule in Prenatally Programmed Hypertension in the Rat. Pediatric Nephrology, 27, 285-293. http://dx.doi.org/10.1007/s00467-011-1976-9

[9] Chou, H.S., Wang, L.F., Lu, K.S. and Chen, C.M. (2008) Effects of Maternal Undernutrition on Renal Angiotensin II and Chymase in Hypertensive Offspring. Acta Histochemica, 110, 497-504. http://dx.doi.org/10.1016/j.acthis.2008.01.002

[10] Yakovleva, T.V., Bazhan, N.M. and Makarova, E.N. (1997) Effects of Food Deprivation in Early Pregnancy on the Development of ovaries and Adrenals in Female Progeny of the Water Vole (Arvicola Terrestris). Comparative Biochemistry and Physiology,116, 103-109. http://dx.doi.org/10.1016/s0742-8413(96)00132-6 
[11] Chavarro, J.E., Rich-Edwards, J.W., Rosner, B.A. and Willett, W.C. (2008) Protein Intake and Ovulatory Infertility. American Journal of Obstetrics \& Gynecology, 19, 210.e1-210.e7. http://dx.doi.org/10.1016/j.ajog.2007.06.057

[12] Hilakivi-Clarke, L., Clarke, R., Onojafe, I., Raygada, M., Cho, E. and Lippman, M. (1997) A Maternal Diet High in n6 Polyunsaturated Fats Alters Mammary Gland Development, Puberty Onset, and Breast Cancer Risk among Female Rat Offspring. Proceedings of the National Academy of Sciences of the United States of America, 94, 9372-9377. http://dx.doi.org/10.1073/pnas.94.17.9372

[13] Hilakivi-Clarke, L., Clarke, R. and Lippman, M. (1999) The Influence of Maternal Diet on Breast Cancer Risk among Female Offspring. Nutrition, 15, 392-401. http://dx.doi.org/10.1016/S0899-9007(99)00029-5

[14] Painter, R.C., De Rooij, S.R., Bossuyt, P.M.M., Osmond, C., Barker, D.J.P., Bleker, O.P. and Roseboom, T.J. (2006) A Possible Link between Prenatal Exposure to Famine and Breast Cancer: A Preliminary Study. American Journal of Human Biology, 18, 853-856. http://dx.doi.org/10.1002/ajhb.20564

[15] Barker, D.J.P., Osmond, C., Thornburg, K.L., Kajantie, E. and Eriksson, J.G. (2008) A Possible Link between the Pubertal Growth of Girls and Ovarian Cancer in Their Daughters. American Journal of Human Biology, 20, 659-662. http://dx.doi.org/10.1002/ajhb.20789

[16] Clutton-Brock, T. (1988) Reproductive Success. 3rd Edition, University of Chicago Press, Chicago.

[17] Meikle, D. and Westberg, M. (2001) Maternal Nutrition and Reproduction of Daughters in Wild House Mice (Mus musculus). Reproduction, 122, 437-442. http://dx.doi.org/10.1530/rep.0.1220437

[18] Pope, W.F. (1988) Uterine Asynchrony-A Cause of Embryonic Loss. Biology of Reproduction, 39, 999-1003. http://dx.doi.org/10.1095/biolreprod39.5.999

[19] Andrews, W.W. and Ojeda, S.R. (1981) A Detailed Analysis of the Serum Luteinizing-Hormone Secretory Profile in Conscious, Free-Moving Female Rats during the Time of Puberty. Endocrinology, 109, 2032-2039. http://dx.doi.org/10.1210/endo-109-6-2032

[20] Wade, G.N. and Schneider, J.E. (1992) Metabolic Fuels and Reproduction in Female Mammals. Neuroscience \& Biobehavioral Reviews, 16, 235-272. http://dx.doi.org/10.1016/S0149-7634(05)80183-6

[21] Bergendahl, M. and Veldhuis, J.D. (1995) Altered Pulsatile Gonadotropin Signaling in Nutritional Deficiency in the Male. Trends in Endocrinology \& Metabolism, 6, 145-159. http://dx.doi.org/10.1016/1043-2760(95)00081-R

[22] Liang, H. and Zhang, Z.B. (2006) Food Restriction Affects Reproduction and Survival of F1 and F2 Offspring of RatLike Hamster (Cricetulus Triton). Physiology \& Behavior, 87, 607-613. http://dx.doi.org/10.1016/j.physbeh.2005.12.006

[23] Rosso, P., Keyou, G., Bassi, J.A. and Slusser, W.M. (1981) Effect of Malnutrition during Pregnancy on the Development of the Mammary Glands of Rats. Journal of Nutrition, 111, 1937-1941

[24] Ergaz, Z., Avgil, M. and Ornoy, A. (2005) Intrauterine Growth Restriction-Etiology and Consequences: What Do We Know about the Human Situation and Experimental Animal Models? Reproductive Toxicology, 20, 301-322. http://dx.doi.org/10.1016/j.reprotox.2005.04.007

[25] McGuire, M.K., Littleton, A.W., Schulze, K.J. and Rasmussen, K.M. (1995) Pre- and Postweaning Food Restrictions Interact to Determine Reproductive Success and Milk Volume in Rats. Journal of Nutrition, 125, 2400-2406.

[26] Rosenfeld, C.S., Grimm, K.M., Livingston, K.A., Brokman, A.M., Lamberson, W.E. and Roberts, R.M. (2003) Striking Variation in the Sex Ratio of Pups Born to Mice According to Whether Maternal Diet Is High in Fat or Carbohydrate. Proceedings of the National Academy of Sciences of the United States of America, 100, 4628-4632. http://dx.doi.org/10.1073/pnas.0330808100 